\title{
Hydrogen absorption and desorption in steel by electrolytic charging
}

\author{
G. Mertens ${ }^{1, a}$, L. Duprez ${ }^{2, b}$, B.C. De Cooman ${ }^{3, c}$, M. Verhaege ${ }^{1}$ \\ ${ }^{1}$ Department of Metallurgy and Material Science, Ghent University, Technologiepark 903, 9052 \\ Zwijnaarde (Gent), Belgium \\ ${ }^{2}$ OCAS (Arcelor Group), J.F. Kennedylaan 3, 9042 Zelzate (Gent), Belgium \\ ${ }^{3}$ Pohang University of Science and Technology \\ a'Geert.Mertens@UGent.be, 'bode.Duprez@arcelor.com, 'decooman@postech.ac.kr
}

Keywords: hydrogen trapping, hydrogen diffusivity, electrolytic hydrogen charging, hydrogen damage

\begin{abstract}
The presence of hydrogen in steel decreases its toughness and formability leading to hydrogen embrittlement. To understand the failure mechanisms of steel due to the presence of hydrogen, a profound insight in the hydrogen household of the steel is needed. This includes a study of the solubility, the diffusion and the trapping of hydrogen. Next, the absorption and desorption behavior during and after electrolytic charging must be well determined. This was investigated in this research for steels with various types of traps, e.g. dislocations, microcracks, grain boundaries and precipitates such as $\mathrm{TiC}$ and $\mathrm{Ti}_{4} \mathrm{C}_{2} \mathrm{~S}_{2}$. The samples were cathodically charged at three different current densities: $0.8 \mathrm{~mA} / \mathrm{cm}^{2} ; 8.3 \mathrm{~mA} / \mathrm{cm}^{2}$ and $62.5 \mathrm{~mA} / \mathrm{cm}^{2}$. It was noticed that the cathodic current density used for hydrogen loading had a great influence on the results. Observation of the samples by scanning electron microscopy (SEM) showed that at the highest current density major damage of the surface had occurred. Hence it was decided to study more systematically the influence of charging current density on the resulting surface aspect and on hydrogen absorption and desorption. The hydrogen charging kinetics, maximum hydrogen solubility and hydrogen desorption behavior have also been evaluated for the different current densities during charging.
\end{abstract}

\section{Introduction}

The presence of hydrogen in steel is reported to be detrimental for its toughness and formability. This phenomenon is called hydrogen embrittlement [1,2,3,4]. Many studies were conducted on the diffusion and trapping of hydrogen in steel $[4,5,6,7,8,9,10]$ and other metals [10]. Hydrogen can be introduced in the steel through cathodic charging. The influence of the thickness and the roughness of the sample, the promoters in the electrolyte, the current density and a heat treatment on the permeation curves in Armco-Fe and AF1410 steel was studied by Mamani [9].

The current density during hydrogen charging was found to have a great influence on the absorption and desorption of hydrogen in steel [9]. However, to study hydrogen-induced phenomena in steel a wide range of current densities are used varying from $0.02 \mathrm{~mA} / \mathrm{cm}^{2}$ to $40 \mathrm{~mA} / \mathrm{cm}^{2}[8,10]$. From the results of hydrogen absorption obtained during and desorption obtained after cathodic charging, the influence of the presence of $\mathrm{H}$-traps of varying strengths was investigated.

\section{Experimental}

\section{Materials}

A selection of cold rolled steels was made in order to investigate the influence of different kinds of hydrogen traps. Annealed ultra low carbon steel (ULC) was used as reference material. Table 1 gives an overview of the materials used with their respective hydrogen trapping sites. 
Overview of the materials, used for the optimization of the equipment, with their different trapping sites

\begin{tabular}{|l||l|l|l|l|l||}
\cline { 2 - 6 } \multicolumn{1}{c|}{} & $\mathrm{C}(\mathrm{ppm})$ & $\mathrm{Mn}(\%)$ & $\mathrm{S}(\mathrm{ppm})$ & $\mathrm{Ti}(\mathrm{ppm})$ & Trapping sites \\
\hline ULC annealed & 26 & 0.15 & - & - & $\begin{array}{l}\text { Grain boundaries, } \\
\text { Dislocations }\end{array}$ \\
\hline ULC 75\% cold rolled & 26 & 0.15 & - & - & Dislocations \\
\hline DC03ED & 30 & $0.1-0.3$ & $<200$ & - & Cracks, voids \\
\hline DC04EK & $200-400$ & $0.2-0.3$ & $<200$ & - & Cracks, cementite \\
\hline DC06EK & $50-100$ & $0.1-0.2$ & $100-300$ & $0.08-0.12$ & Precipitates (TiC, MnS) \\
\hline
\end{tabular}

Table 1

An overview of the microstructure of the materials in Table 1 is given through Fig. 1.

(a)

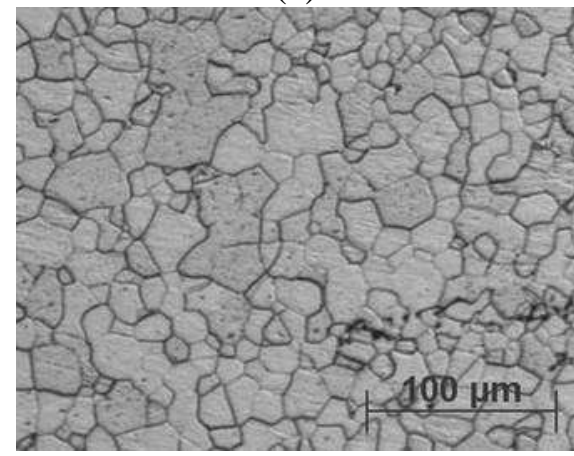

(d)

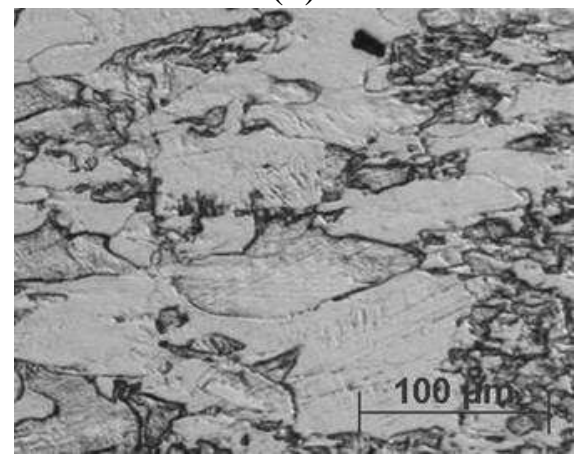

(b)

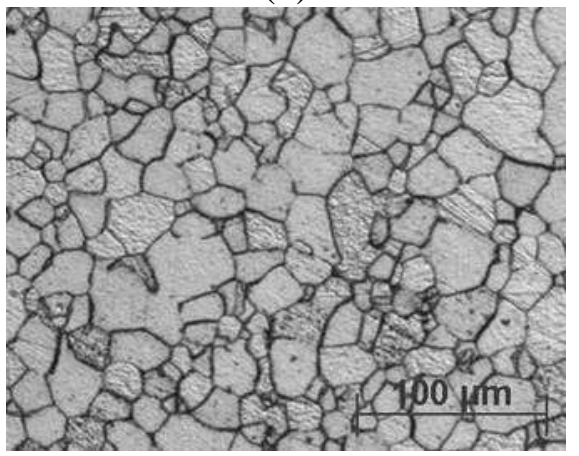

(e)

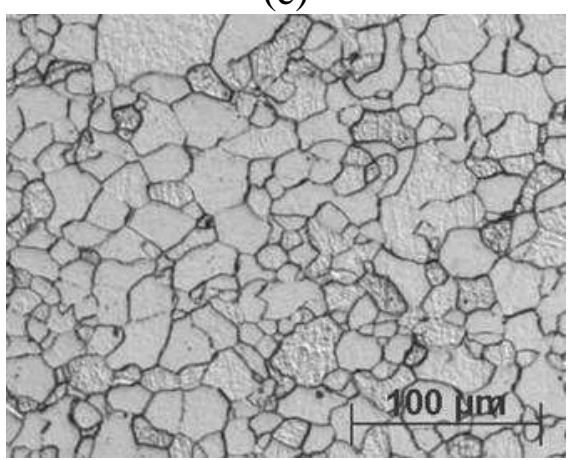

(c)
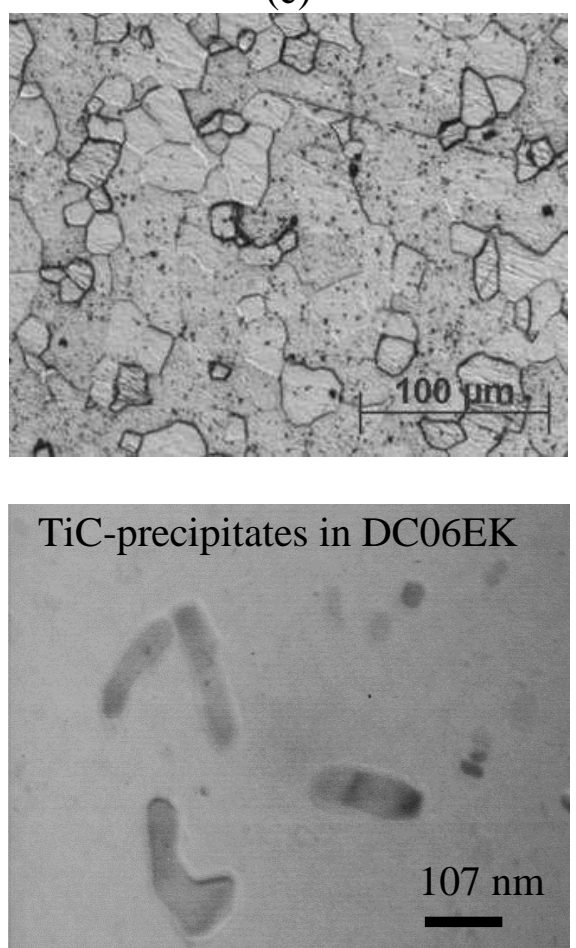

Fig. 1: Initial microstructure of the materials used: (a) DCO3ED, (b) DC04EK, (c) DCO6EK with a TEM observation of TiC precipitates in DC06EK, (d) ULC $75 \%$ cold rolled, (e) ULC annealed

All samples, with a thickness of $1 \mathrm{~mm}$, were cut into a rectangular shape of $6 \mathrm{~mm} \times 10 \mathrm{~mm}$ and were stored in isopropanol to avoid hydrogen uptake from the environment and corrosion. The samples were used as received because preliminary experiments showed that no hydrogen uptake, i.e. absorption or adsorption occurred during mechanical polishing. Before each measurement the samples were ultrasonically cleaned in acetone for at least 15 minutes.

\section{Methods}

All samples were cathodically charged, at a selected constant current, in an aqueous solution with the same composition as the one which is used in the standardized Ströhlein test EN10209 (6vol\% $\mathrm{H}_{2} \mathrm{SO}_{4}, 0.25 \mathrm{~g} / 1 \mathrm{HgCl}_{2}$ and $0.5 \mathrm{~g} / 1 \mathrm{As}_{2} \mathrm{O}_{3}$ ). The anode was a Pt wire. The amount of hydrogen in the sample was measured as a function of charging time at different current densities: $62.5 \mathrm{~mA} / \mathrm{cm}^{2}$, $8.3 \mathrm{~mA} / \mathrm{cm}^{2}$ and $0.8 \mathrm{~mA} / \mathrm{cm}^{2}$. This was done by combustion of the sample in a Ströhlein H-Mat 2500 analyzer. The same apparatus was used to analyze hydrogen after different discharge times. The samples were kept in isopropanol during discharge. The damage of the samples has been characterised by means of SEM (Carl Zeiss DSM 962). 


\section{Results and discussion.}

The influence of the current density on the absorption kinetics and damage of the material The influence of the current density on hydrogen charging characteristics of the samples mentioned in Table 1 is shown in Fig. 2. It can be seen that for all materials higher current densities result in faster initial absorption kinetics and a higher amount of hydrogen after charging until steady state. The latter can be related to the increasing damage of the material when higher current densities during charging are used, as shown in Fig. 3 for DC03ED. Because hydrogen tends to be attracted by regions of high triaxial tensile stress where the metal structure is dilated, cracks will act as hydrogen traps. Due to this, the accumulation of hydrogen occurs at those sites which results in an increased internal pressure on the crack and promotes its propagation. Hence, a high current density results in an increased damage leading to additional trapping sites (cracks) and higher hydrogen content. It should be noticed that in most cases steady state is reached after a certain time of charge. This suggests that from this moment on no further damage takes place in the sample. Further investigation of this phenomenon will be undertaken.

Because the hydrogen absorption of DC03ED was found to proceed in a similar way as DC04EK, only the latter is shown in Fig. 2.
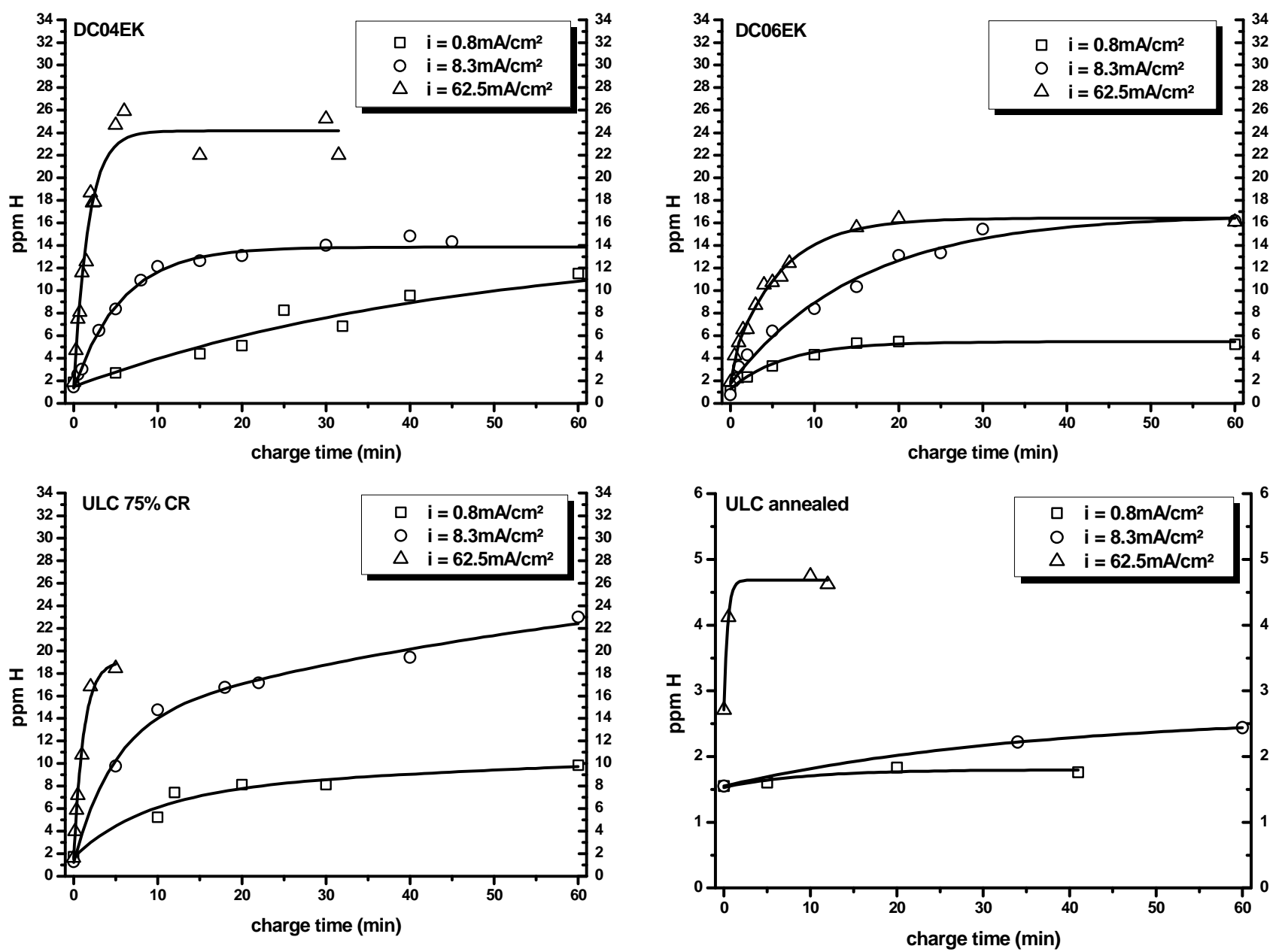

Fig. 2 Absorption kinetic for the steel grades as a function of the charge current density. 
(a)

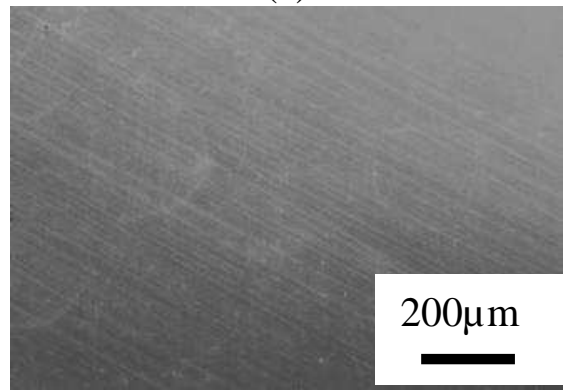

(b)

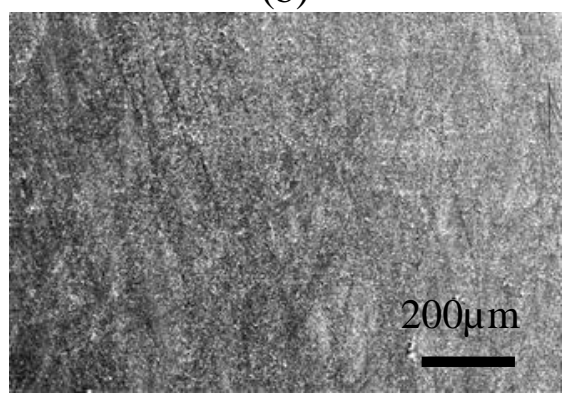

(c)

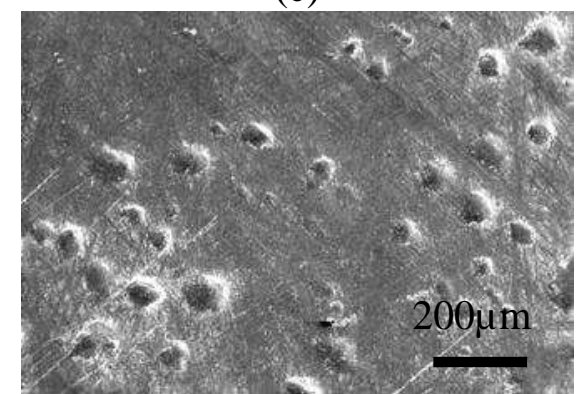

Fig. 3 SEM observation of the increasing damage at the surface of DC03ED with increasing current densities: (a) not charged, $(b) \mathrm{i}=0.8 \mathrm{~mA} / \mathrm{cm}^{2}$ and $(c) \mathrm{i}=62.5 \mathrm{~mA} / \mathrm{cm}^{2}$.

Also depending on the charge current density is the time of charge needed to reach steady state. In Fig. 2, it can be seen that the time needed to reach steady state is inversely proportional to charge current density for all materials.

Fig. 4 shows the absorption kinetics for the different materials at a low current density and at a high current density. For the high as well as for the low current density, the lowest content of hydrogen after charging until steady state and the slowest absorption rate is found in the annealed ULC material, where no traps are present and almost no damage occurred. It is worth to notice that after cold rolling, the steady state value of hydrogen absorption increases from $1.8 \mathrm{ppm}$ to $10 \mathrm{ppm}$ at a current density of $0.8 \mathrm{~mA} / \mathrm{cm}^{2}$ and from $5 \mathrm{ppm}$ to $17 \mathrm{ppm}$ at a current density of $62.5 \mathrm{~mA} / \mathrm{cm}^{2}$. This indicates that the presence of dislocations in the steel enhances the sensibility to damage during hydrogen charging. Fig. 4 also shows that DC06EK has an intermediate level of hydrogen absorption for the high as well for the low current density. This suggests that the sensitivity to damage during hydrogen charging depends on the kind of trapping sites in the material.

On the other hand, from the same figure it is also obvious that no distinction related to the trapping capacities between the studied materials can be made suggesting that the current densities used are too high to study the trapping phenomena in steel. This is also confirmed in Fig. 3 for DC03ED and in Fig. 5 for the other materials, where blisters at the surface are observed. The presence of blisters at the surface can be related to an increased internal pressure, due to hydrogen recombination or hydrogen accumulation, in the bulk of the material.
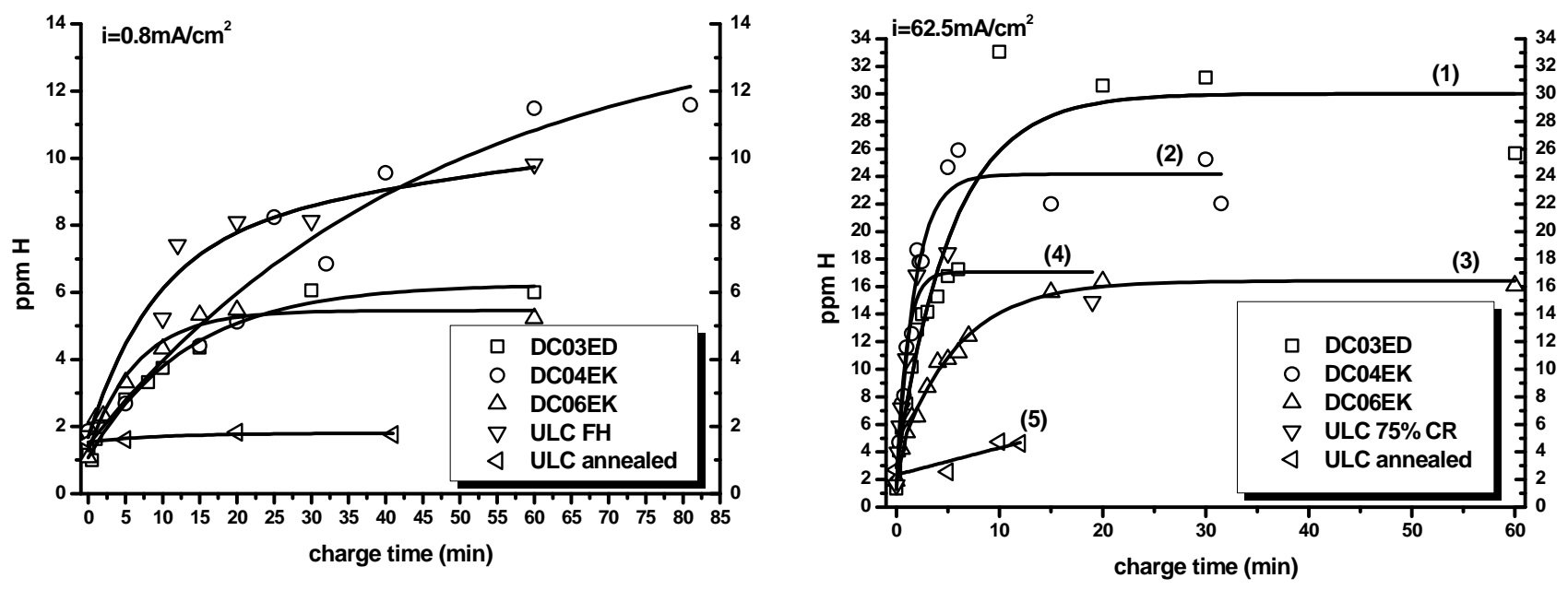

Fig. 4 Overview of the absorption kinetics for the materials used at a constant current density 
(a)

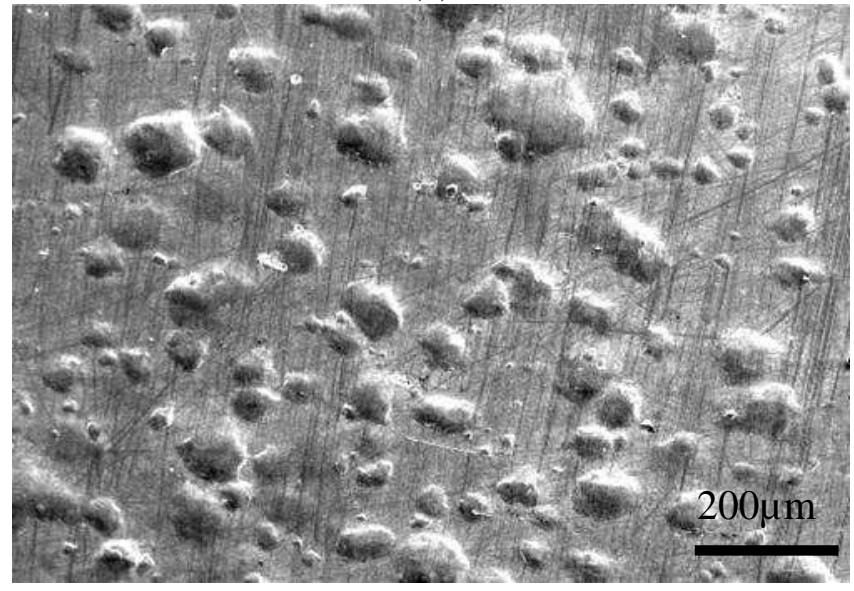

(c)

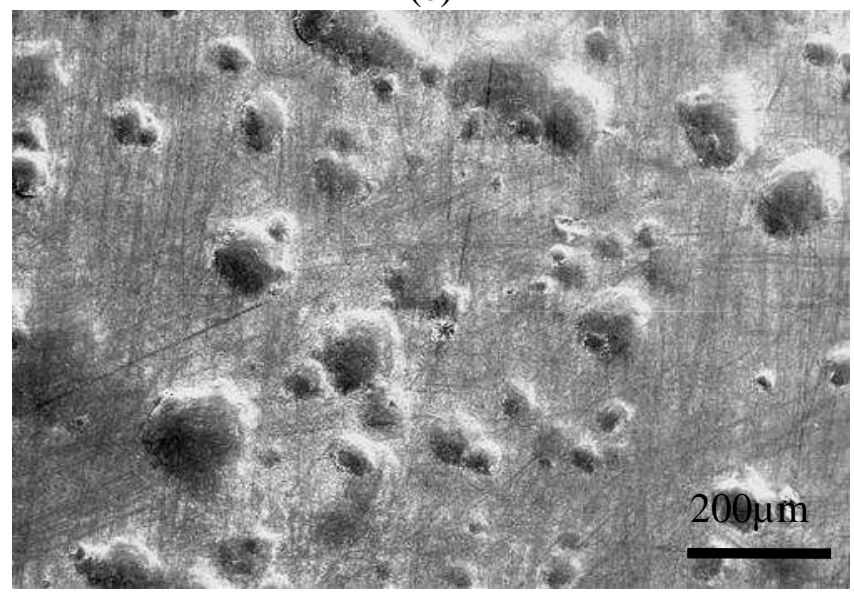

(b)

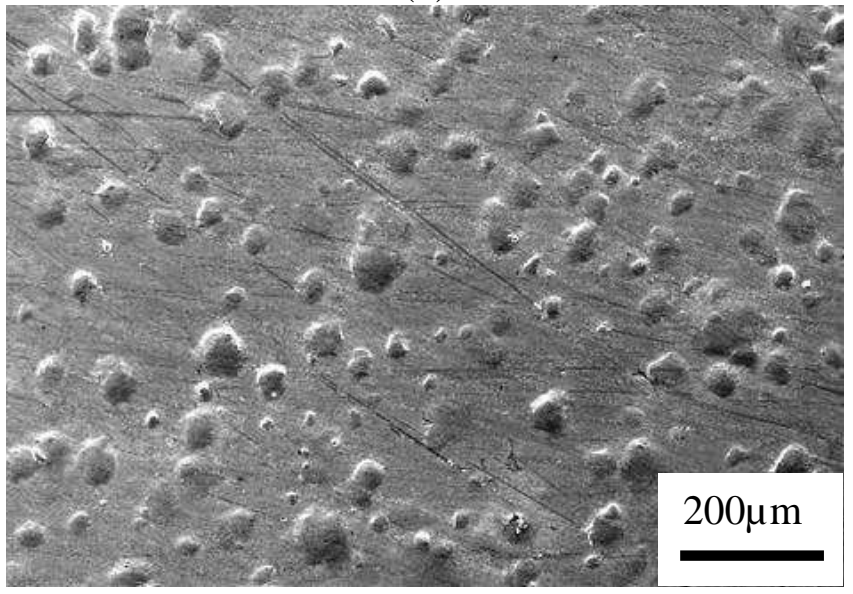

(d)

Fig. 5: SEM-observations of the surface of the materials used (a) DC04EK, (b) DC06EK, (c)ULC cold rolled and (d) ULC annealed, after cathodically charging for 6 minutes at a current density of $62.5 \mathrm{~mA} / \mathrm{cm}^{2}$.

\section{Desorption kinetics}

Fig. 6 shows the kinetics of hydrogen desorption for the materials under investigation after charging for 6 minutes at a current density of $62.5 \mathrm{~mA} / \mathrm{cm}^{2}$. It can be seen that cold rolled ULC shows a high initial desorption rate indicating that hydrogen escapes from the dislocations very fast. This corresponds with the fact that dislocations are reversible, weak hydrogen trapping sites. After two hours, however, the desorption kinetics of the cold rolled ULC steel become slower showing that the hydrogen is irreversibly trapped in the cracks of the damaged material.

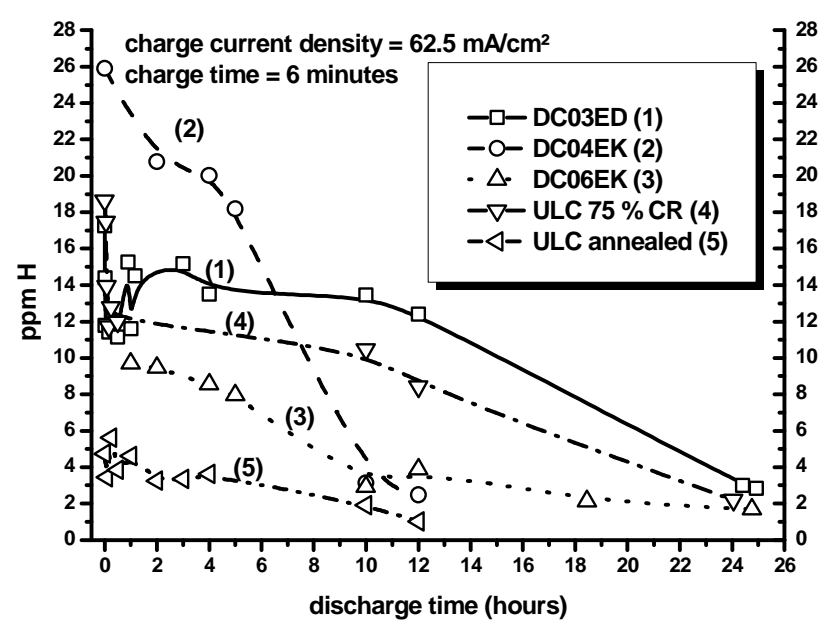

Fig. 6 Desorption kinetics of the materials used after charging for 6 minutes and $i_{c}=62.5 \mathrm{~mA} / \mathrm{cm}^{2}$ 


\section{Conclusions}

The charging kinetics differ strongly depending on the current density used during cathodic hydrogen charging and on the microstructure of the material. As seen in Fig. 3 increasing the current density results in an increasing damage of the surface. The mechanism can be described by the recombination of hydrogen at the trapping sites of the material generating blisters at the surface of the charged material. This change of the surface aspect of the material influences the absorption kinetics.

Higher hydrogen absorption kinetics and a higher hydrogen content after charging are expected when dislocations are present after cold rolling of annealed ULC. The presence of $\mathrm{TiC}$ and $\mathrm{Ti}_{2} \mathrm{C}_{4} \mathrm{~S}_{4}$ shows intermediate absorption kinetics and hydrogen content. This suggests that the resistance of the material to damage during cathodic hydrogen charging depends on the kind of its trapping sites.

To study the trapping capacity of steel, lower current densities than $0.8 \mathrm{~mA} / \mathrm{cm}^{2}$ should be applied to avoid damage of the material. Under this condition, the maximum hydrogen level after charging until steady state can be an indication of the amount of trapping sites in the steel.

Charging to steady state at a current density where the material is not damaged will enable us to calculate then the strength of the trapping sites from the desorption kinetics of the material.

In later studies, a more profound study of the damage will be established. Charging at a certain current density during varying charge times will enable us to characterize the damage. We expect that at the same moment, when steady state occurs in the absorption curve, the damage of the material will stop. On the other hand, a critical charge current density has to be defined where no damage will occur.

\section{References}

[1] X. Wu, Y. Katada, S.G. Lee, I.S. Kim: Hydrogen-Involved Tensile and Cyclic Deformation Behavior of Low-Alloy Pressure Vessel Steel Metall. and Mat. Trans. A, vol. 35A, May (2004), pp.1477-1486

[2] J.S. Lee, A. Kimura, S. Ukai, M. Fujiwara: Effects of hydrogen on the mechanical properties of oxide dispersion strengthening steels Journal of Nuclear Materials 329-333 (2004), pp. 1122-1126

[3] D. Hardie, J. Xu, E.A. Charles, Y. Wei: Hydrogen embrittlement of stainless steel overlay materials for hydrogenators Corrosion Science 46 (2004), pp. 3089-3100

[4] R.A. Oriani: The Physical and Metallurgical Aspects of Hydrogen in Metals Fourth International Conference on Cold Fusion (1993)

[5] K. Kiuchi, R.B. McLellan: The solubility and diffusivity of hydrogen in well-annealed and deformed iron Acta metall., vol. 31, no. 7 (1983), pp. 961-984

[6] H. Hagi: Diffusion Coefficient of Hydrogen in Iron without Trapping by Dislocations and Impurities Mat. Trans. JIM, vol. 35, no. 2 (1994), pp. 112-117

[7] J.P. Hirth: Effects of Hydrogen on the Properties of Iron and Steel Metall. Trans. A, vol. 11A, June (1980), pp. 861-890

[8] H. Asahi, D. Hirakami, S. Yamasaki: Hydrogen Trapping Behavior in Vanadium-added Steel ISIJ International, vol. 43 (2003), no. 4, pp. 527-533

[9] S.C. Mamani: Study of Hydrogen Permeation and Diffusion in Steels: Predictive Model for Determination of Desorbed Hydrogen Concentration University of Puerto Rico, Mayagüez Campus (2005)

[10]M.D. Danford: Hydrogen Trapping and the Interaction of Hydrogen With Metals NASA Technical Paper 2744 (1987) 